\title{
Estado Epiléptico No Convulsivo Infantil
}

\author{
JUAN ARÁNGUIZ R. ${ }^{1}$, MARTA HERNÁNDEZ CH. ${ }^{2}$, RAÚL ESCOBAR H. ${ }^{2}$, TOMAS MESA L. ${ }^{2}$ \\ 1. Residente de Neurología Pediátrica, Departamento de Pediatria, Pontificia Universidad Católica de Chile-Santiago. \\ 2. Neurólogo Infantil Departamento de Pediatría, Unidad de Neurología Infantil, Departamento de Pediatría, \\ Pontificia Universidad Católica de Chile-Santiago.
}

\begin{abstract}
Non Convulsive Status Epilepticus in Children

Introduction: Status Epilepticus (SE) is a medical emergency with high morbimortality. Non-convulsive Status Epilepticus (NCSE) is defined as electroencephalographic crises in the absence of clinical motor symptoms. Objective: A restrospective study of NCSE in our population. Patients and Methods: Charts of 30 patients in a Child Neurology Clinic seen between December 1999 and June 2008 were reviewed. According to the characteristics of the episode, they were classified as a) Typical absence, b) Atypical absence, c) Partial Complex episode. Results: Of the 30 patients, 15 (50\%) were males. Median age was 46 months. Clinically, $63 \%$ of the children suffered from complex partial seizures, $30 \%$ atypical absences, $7 \%$ typical absences. Cryptogenic SE was most frequent (47\%), with a mortality of 3\%. Discussion: Pediatric patients have a higher risk of NCSE than adults do. Most patients already carried a diagnosis of Epilepsy, cerebral infarcts were the second most frequent cause of NCSE. They should all be monitored through EEGs. Neuroimages are of great value since outcome depends on the etiology. In summary, clinical suspicion, a history of epilepsy supported by an EEG (best if prolonged) allow early diagnosis and treatment.

(Key words: Non-convulsive Status Epilepticus, children, Status Epilepticus, electroencephalogram, Cryptogenic status).

Rev Chil Pediatr 2010; 81 (2): 115-122

\section{RESUMEN}

Introducción: El Estado Epiléptico (EE) es una emergencia médica con alta morbimortalidad. El Estado Epiléptico No Convulsivo (EENC) es definido por la presencia de crisis electroencefalográficas en ausencia de crisis motoras clínicas. Objetivo: Realizar una descripción retrospectiva de los EENC que se presentaron en la población pediátrica atendida en nuestro centro. Pacientes y Método: Se revisaron los registros de 30 pacientes controlados neurología infantil que presentaron EENC entre diciembre de 1999 y junio de 2008. Según el tipo de crisis se clasificó en a) EENC ausencia típica; b) EENC ausencias atípicas y c) EENC parcial complejo. Según etiología se uso la clasificación de Hauser modificada. Resultados: Se analizaron 30 pacientes. Quince (50\%) varones. La mediana de edad fue 46 meses. 63\% fueron EENC parcial complejo, 30\% EENC ausencias atípicas y 7\% EENC ausencias típicas. El EE criptogénico fue el más frecuente (47\%). La
\end{abstract}

Trabajo recibido el 03 de noviembre de 2009, devuelto para corregir el 07 de diciembre de 2009, segunda versión el 05 de enero de 2010, aceptado para publicación el 23 de enero de 2010.

Correspondencia a:

Juan L. Aránguiz R.

E-mail: juanluis.aranguiz@gmail.com 
mortalidad fue de 3\%. Discusión: Los pacientes pediátricos tienen mayor riesgo de EENC en relación a los adultos. La gran mayoría de los pacientes con EENC ya tenían el diagnóstico de epilepsia. Los infartos cerebrales fueron la segunda causa de EENC, estos deben ser monitorizados con EEG ante la sospecha de EENC. Las neuroimágenes para precisar el diagnóstico son de gran valor dado que el pronóstico de los pacientes con EENC depende de la etiología subyacente. En conclusión, la sospecha clínica, el antecedente de epilepsia y el apoyo con un EEG, especialmente prolongado, permite el diagnóstico oportuno y el tratamiento precoz.

(Palabras clave: Estado Epiléptico No Convulsivo, etiología, niños, clasificación, pronóstico, monitoreo electroencefalográfico continuo).

Rev Chil Pediatr 2010; 81 (2): 115-122

\section{Introducción}

El Estado Epiléptico (EE) es una emergencia médica y neurológica. Tiene asociada una morbilidad y mortalidad significativa a pesar de los tratamientos existentes por lo que su diagnóstico y manejo debe ser adecuado y oportuno $^{1}$. La definición convencional de estado epiléptico corresponde a crisis epilépticas continuas de más de 30 minutos de duración o crisis recurrentes, sin recuperación completa de la función neurológica entre los eventos ${ }^{2-4}$. Existe discusión entre algunos autores acerca de la definición de Estado Epileptico No Convulsivo (EENC), pero en marzo del 2004, en Oxford, en el Research Foundation Workshop sobre EENC se propuso la siguiente definición: "EENC es el término usado para describir un rango de condiciones en las cuales la actividad electroncefalográfica es prolongada y resulta en síntomas clínicos no convulsivos".

Desde un punto de vista operacional se considera un mínimo de tiempo, usualmente 30 minutos de actividad electrográfica continua o recurrente, responsable de síntomas clínicos variables. Se debe reconocer que este es un tiempo arbitrario.

Los criterios electroencefalográficos se clasifican en criterios definitivos y criterios equívocos. Los criterios definitivos en el EEG son:

a) Frecuentes o continuas crisis electrográficas focales, con patrón ictal tipo espigaonda con cambios en amplitud, frecuencia y/o distribución espacial.

b) Frecuentes o continuas descargas espigaonda, en pacientes sin historia previa de epilepsia. c) Frecuentes o continuas descargas espigaonda generalizadas con evolutividad cuando se comparan con la basal, en pacientes epilépticos.

d) PLEDs (descargas epileptiformes lateralizadas periódicas) o biPEDs (descargas epileptiformes periódicas bilaterales) que ocurren en pacientes en coma.

\section{Los criterios equívocos son:}

a) Frecuentes o continuas anormalidades del EEG (espigas, espiga-onda, actividad rítmica lenta, PLEDs, biPEDs, ondas trifásicas) en pacientes que previamente no mostraron estos hallazgos, en el contexto de un daño cerebral agudo (por ejemplo daño hipóxico, infección, trauma, etc).

b) Frecuentes o continuas anormalidades, generalizadas, en el EEG en pacientes con encefalopatía epiléptica en los cuales se ha visto un patrón interictal similar pero cuyos síntomas sugieren un EENC.

Los pacientes con EENC pueden presentar una amplia variedad de manifestaciones clínicas incluyendo coma, confusión, somnolencia, alteración del ánimo, estados de fuga, afasia, síntomas autonómicos o vegetativos anormales, alucinaciones y paranoia ${ }^{5,6,13}$. Todos estos síntomas deben aparecer en ausencia de actividad convulsiva (tónica, clónica o tonicoclónica) evidente. No obstante, pueden observarse manifestaciones motoras sutiles, como mioclonías palpebrales, automatismos manuales u oroalimentarios, giros versivos de la cabeza, discretas posturas distónicas de las extremidades o nistagmo. 
Clásicamente, se distinguen dos tipos principales de EENC según los cambios electroencefalográficos ictales ${ }^{6-9}$ :

1. EENC generalizado y/o de ausencia.

2. EENC parcial.

Además, la presencia de afectación o no de la conciencia durante el episodio permite distinguir dos subtipos de EENC parcial:

1. EE parcial simple (EEPS): sin afectación de la conciencia (muy poco frecuente).

2. EE parcial complejo (EEPC): con afectación de la conciencia.

El EENC suele ser subdiagnosticado o diagnosticado tardíamente ya que requiere de un alto índice de sospecha y la realización de un electroencefalograma ${ }^{6,10,11}$. La mayoría de los EENC se presentan en pacientes a los cuales supuestamente se les controló un Estado Epiléptico Convulsivo, pero que persisten con compromiso de conciencia cualitativo o cuantitativo.

Diversas series han demostrado 23 a $34 \%$ de EENC en niños sometidos a monitoreo EEG en unidades de cuidados intensivos ${ }^{12-15}$. El diagnóstico de EENC es importante porque tiene asociada un aumento de la morbilidad neurológica y secuelas a largo plazo ${ }^{11,16-18}$. El tratamiento con drogas antiepilépticas tiene buenos resultados, dependiendo de la etiología.

El objetivo de este estudio es describir las características clínicas y electroencefalográficas de pacientes pediátricos con EENC que acudieron al Hospital Clínico de la Universidad Católica (PUC) entre diciembre de 1999 y junio de 2008.

\section{Pacientes y Método}

Se revisaron los registros de Estado Epiléptico de pacientes controlados en neurología infantil en la red de salud de la PUC y que presentaron un EENC. Durante el período comprendido entre el 1 de diciembre de 1999 al 30 de junio de 2008. En este estudio se excluyeron recién nacidos y pacientes cursando con una encefalopatía epiléptica y a aquellos que recibieron paralizantes que expliquen la disociación electroclínica.

Se definió EENC como aquel EE que se presentó como compromiso de conciencia o neurológico sin manifestaciones motoras y que se acompañó de un electroencefalograma (EEG) ictal (descargas frecuentes o continuas, focales o generalizadas, evolutivas, de tipo espiga-onda o punta-onda, theta-delta rítmico, poli espigas, en pacientes con o sin historia previa de epilepsia y descargas epileptiformes lateralizadas periódicas en pacientes en coma, en relación a un EE tónico-clónico generalizado).

Se definió como ausencia tipica la presentada en niños o adolescentes, neurológica y mentalmente normales, con epilepsia generalizada idiopatica con crisis de ausencia. Con una alteración muy variable del nivel de conciencia con automatismos como parpadeo rítmico, clonías faciales, mandibulares o de las extremidades. En el EEG se observa una actividad continua o recurrente de descargas epileptiformes generalizadas de punta-onda o polipuntaonda a 3-4 Hz. Las ausencias atípicas se observan en pacientes con epilepsia generalizada sintomática o criptogénica. A menudo sufren retraso mental y, por tanto, puede ser difícil identificar un cambio significativo de su comportamiento basal, los padres o familiares más cercanos suelen advertir que el niño está más hipotónico, menos reactivo o participativo de lo habitual. En el EEG se evidencian descargas generalizadas continuas de complejos puntaonda lenta de 2,0-2,5 Hz, que abarcan la práctica totalidad del registro (al menos más del $55 \%$ ), y se asocia a modificaciones patentes del comportamiento. La alteración de la conciencia suele ser más grave que en el estado de ausencia típico y los automatismos, atonía y mioclonías más prominentes 9 .

El diagnóstico de EENC se realizó con un electroencefalograma compatible con EE y/o monitoreo electroencefalográfico continuo, en pacientes comprometidos de conciencia y sin actividad motora. El EEG se practicó precozmente a todos los pacientes en los que se sospechó el diagnóstico. El monitoreo electroencefalográfico se hizo a aquellos pacientes en que se sospechaba EENC, porque presentaban un 
compromiso de conciencia o habían sido tratados por un EEC y no había recuperación de conciencia después del manejo. Consistió en un monitoreo electroencefalográfico de al menos 4 horas con o sin video. El equipo digital usado fue un Cadwell Easy 2.1 ó un Profile Medelec Oxford.

Se incluyó a todos los pacientes que presentaron un EENC y que tenían entre 1 mes y 15 años de edad a la fecha de presentación.

Todos los pacientes fueron estudiados y tratados en la unidad de paciente crítico pediátrico de nuestra universidad (UPC) y se les realizó además una neuroimagen durante la hospitalización.

Las secuelas neurológicas fueron determinadas por examen neurológico formal. La función cognitiva fue evaluada por examen mental como parte del examen neurológico.

Se registraron los datos demográficos, el tipo de anomalía en el EEG y la etiología, la duración del evento, el estudio electroencefalográfico, neuroimagen, la presencia de recurrencias y la evolución clínica de los pacientes. El seguimiento se efectuó con un rango de 0,5 años a 8 años.

Se clasificaron según etiología usando la clasificación de Hauser $^{19}$ (tabla 1) y según el tipo de crisis en ausencias típica, ausencias atípicas, crisis parciales y parciales complejas ${ }^{4,9}$.

\section{Resultados}

Se completaron los datos de 30 pacientes con EENC hospitalizados en la Unidad de Paciente Crítico del Hospital Clínico de la Universidad Católica de Chile.

\section{Distribución de pacientes por edad y sexo}

E1 50\% $(\mathrm{n}=15)$ hombres. La mediana de edad a la presentación de EE fue de 46 meses (rango de 1,7 a 177 meses), con un promedio de 60,25 meses. Once pacientes $(37 \%)$ tenían menos de dos años de edad, 6 pacientes tenían entre 2 años y 4 años (20\%), mientras 13 pacientes tenían más de 4 años (43\%). A 13 pacientes se les realizó monitoreo EEG.

\section{Duración del EENC}

El tiempo de duración de las crisis varió entre 30 minutos y 49 días hasta el diagnóstico e instauración del tratamiento efectivo. Este último correspondió a un EENC diagnosticado durante el estudio de una alteración de conducta por medio de un monitoreo EEG en un paciente con una displasia cortical bilateral. Excluyendo a este último paciente, la duración promedio de las crisis fue de 95,9 horas desde el inicio de la crisis hasta su término, con una mediana de 30 horas, de menos de 24 horas desde el inicio de la crisis y el diagnóstico en

Tabla 1. Clasificación del Estado Epiléptico según etiología (Hauser modificada)

\begin{tabular}{|ll|}
\hline Tipo & Definición \\
\hline Sintomático agudo & $\begin{array}{l}\text { EE que ocurre durante una enfermedad aguda en la cual hay una noxa neuro- } \\
\text { lógica conocida o una disfunción metabólica sistémica }\end{array}$ \\
\hline Sintomático remoto & $\begin{array}{l}\text { EE que ocurre sin provocación aguda en un paciente con historia de daño del } \\
\text { Sistema Nervioso Central o malformaciones cerebrales }\end{array}$ \\
\hline Sintomático remoto con precipitante agudo & $\begin{array}{l}\text { EE que ocurre en un paciente con encefalopatía crónica, pero con una causa } \\
\text { aguda que lo provoque }\end{array}$ \\
\hline Encefalopatía progresiva & EE que ocurre en un paciente con un trastorno progresivo del SNC \\
\hline Criptogénico & EE que ocurre provocada sólo por fiebre, habiendo excluido una infección del \\
\hline
\end{tabular}

Hauser, W.A., Status epilepticus: epidemiologic considerations. Neurology 1990; 40 (5 Suppl 2): 9-13. 
13 de 15 pacientes de quienes se analizó dicha información.

\section{Forma de presentación de EENC y tipo de descargas en el EEG}

La forma de presentación de estos EENC fue, 19 crisis parciales complejas, 9 ausencias atípicas y 2 ausencias típicas.
El EEG demostró descargas focales en 50\% de los pacientes (15/30), descargas multifocales en $36 \%(11 / 30)$, y descargas generalizadas en $14 \%(4 / 30)$. La morfología de estos registros consistió en descargas frecuentes o continuas, generalizadas o focales, evolutivas, de tipo espiga-onda o punta-onda, theta-delta rítmica y/o poli espigas (figuras 1 y 2 ).

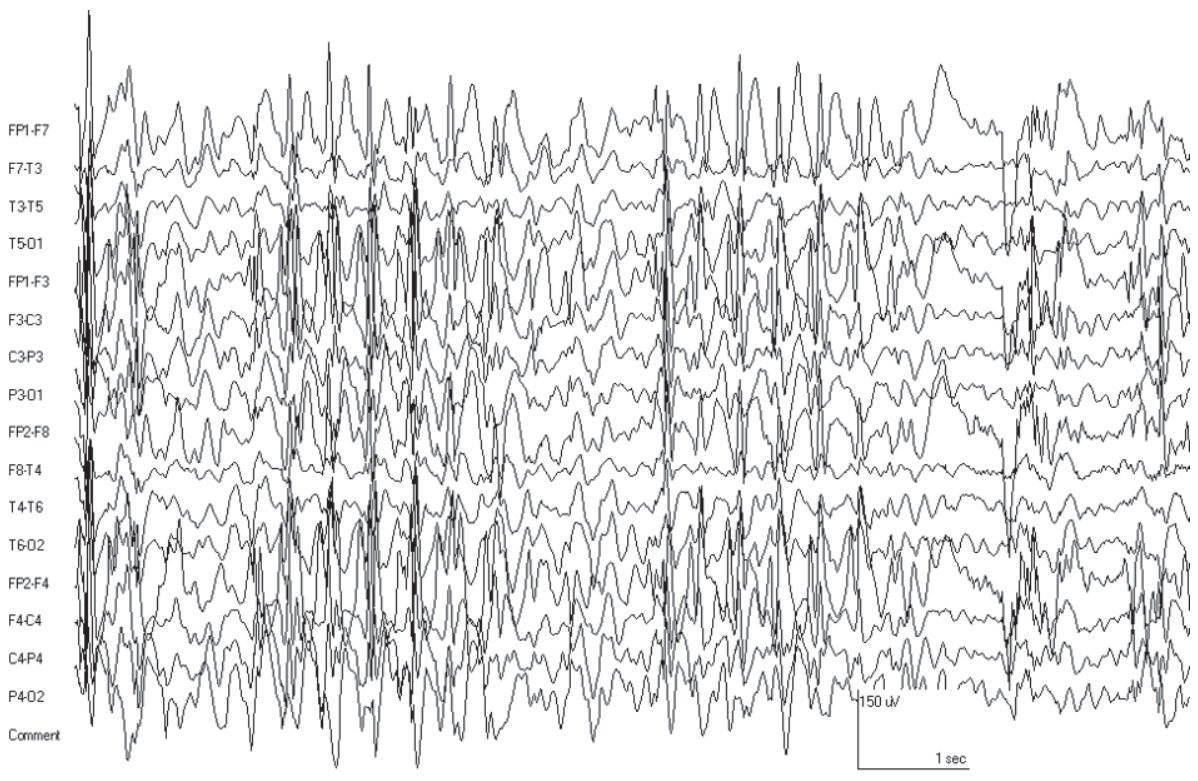

FP1.77 C7.73
Figura 1. EEG que muestra un EENC generalizado criptogénico, en una paciente de 13 años con antecedentes de epilepsia criptogénica en tratamiento.
Figura 2. EEG que muestra un EENC focal (hemisférico izquierdo) gatillado por fiebre, en
un paciente de 7 meses. 


\section{Tipo de EE según etiología:}

Destaca el EE criptogénico (C) como la etiología más frecuente 47\% (14/30). El resto de las etiologías en orden decrecientes son: EE sintomático agudo con 8 (27\%) pacientes, EE sintomático remoto $16 \%(5 / 30)$ y EE sintomático remoto con precipitante agudo en $10 \%$ $(3 / 30)$.

El 73,3\% (22/30) de los pacientes presentaba una epilepsia previa y estaba con tratamiento antiepiléptico al momento de presentación del EENC, de los cuales 45\% (10/22) fue un EENC criptogénico.

\section{Neuroimagen}

Se realizó estudio de imágenes al 100\% de los pacientes, ya sea Tomografía computada (7\%) o Resonancia Nuclear Magnética (93\%). Destaca que 17 niños $(55,5 \%)$ presentaban alguna alteración (tabla 2).

\section{Mortalidad}

Sólo se registró un deceso durante la hospitalización (3\%). Correspondió a un EENC con etiología sintomática remota, a la edad de 12 años 5 meses, quien presentaba secuelas de encefalopatía hipóxico-isquémica y evolucionó con una falla respiratoria grave.

\section{Desarrollo neurológico posterior}

La evolución neurológica se registró sólo en pacientes previamente sanos $(n=11)$. En 19 pacientes con alteraciones neurológicas de

Tabla 2. Hallazgos en la neuroimagen según etiología

\begin{tabular}{|c|c|}
\hline $\begin{array}{l}\text { Clasificación } \\
\text { etiológica }\end{array}$ & Hallazgos neuroimagen \\
\hline $\begin{array}{l}\text { EE Sintomático } \\
\text { agudo }\end{array}$ & $\begin{array}{l}\text { Infartos (6) } \\
\text { Tumor temporoparietal (1) } \\
\text { Leucoencefalopatía (1) }\end{array}$ \\
\hline $\begin{array}{l}\text { EE Sintomático } \\
\text { Remoto + EE } \\
\text { Sintomático } \\
\text { Remoto con } \\
\text { Precipitante } \\
\text { Agudo }\end{array}$ & $\begin{array}{l}\text { Atrofia cerebral (1) } \\
\text { Trastorno de la migración neuronal (3) } \\
\text { Agenesia del cuerpo calloso (1) } \\
\text { Hidrocefalia (1) } \\
\text { Secuela de resección tumoral (1) } \\
\text { Infartos antiguos (2) }\end{array}$ \\
\hline EE Criptogénico & Normal (13) \\
\hline
\end{tabular}

Entre paréntesis el número de casos que presentan esos hallazgos. base esta no se pudo realizar ya que no había una clara evaluación de déficit previos. Se consigna en los controles posteriores que 6 pacientes evoluciona con un desarrollo neurológico anormal (2 pacientes presentan epilepsia, uno de ellos asociado a una parálisis cerebral, 3 pacientes con retraso del desarrollo psicomotor y 1 paciente sólo con parálisis cerebral), de estos pacientes 3 presentaron un EENC criptogénico, 2 secundarios a una encefalitis herpética y 1 a un evento hipóxico isquémico, todos menores de 5 años.

\section{Discusión}

Los pacientes pediátricos tienen un mayor riesgo de EENC que los pacientes adultos, $36 \%$ vs $17 \%$ en el trabajo de Claassen ${ }^{20}$.

En nuestro estudio $37 \%$ fue menor de 2 años, excluyendo neonatos, de los cuales $23,3 \%$ correspondieron a menores de 6 meses. Otros trabajos en niños con alteración de conciencia, $39 \%$ de estos con EENC fueron menores de 6 meses $^{21}$.

En esta serie de pacientes se muestra que el EENC ocurre en niños con y sin historia de epilepsia, pero en su mayoría $(73 \%)$ corresponden a los primeros, similar a lo descrito por Abend, en cuya serie alcanza a $75 \%{ }^{22}$.

La mayoría de los EENC se detectaron alrededor de 24 horas de iniciado los síntomas, destacando la importancia de un registro de más de 30 minutos de duración en aquellos pacientes en que se sospecha un EENC ${ }^{14,22}$, para evitar un diagnóstico tardío como en nuestro paciente que se prolongo por 49 días.

A diferencia de lo descrito por Alroughani la etiología más común en nuestra serie correspondió a EENC criptogénico. Esto probablemente se explica por la población de pacientes epilépticos adultos en la serie de Alroughani. Cabe destacar que la segunda etiología más frecuente fue la causa sintomática aguda y entre ellos los accidentes cerebrovasculares isquemicos infartos, la literatura destaca que los pacientes con infarto cerebral y encefalopatía hipóxico-isquémica tienen 45,2\% de EENC, por lo que es necesario estar atento a dicha complicación en aquellos pacientes ${ }^{23}$. 
Los EENC pueden presentar descargas focales, multifocales o generalizadas. En este trabajo la mayoría correspondió a descargas focales y multifocales (86\%). Resultado similar al observado en otros estudios ${ }^{12,13,21}$. Ningún paciente presentó descargas epileptiformes lateralizadas periódicas.

Estudios previos demostraron una alta incidencia de EENC en un amplio rango de edades y diversas etiologías ${ }^{12,13,20}$. En nuestra serie, el 90\% de los niños menores de 24 meses que presentaron con EENC no tenían historia previa de convulsiones o epilepsia, el total de ellos correspondió a EE sintomático agudo y sólo 1 tenía historia de crisis convulsivas afebriles.

Las etiologías pueden variar según el grupo etáreo, por ejemplo ningún niño menor de 1 año tuvo historia de epilepsia pero destacan en ellos infecciones, encefalopatía hipóxicoisquémica y alteraciones estructurales del sistema nervioso central.

En estudios previos la etiología incluyó encefalopatía hipóxico isquémica en $26 \%$, enfermedad metabólica en $21 \%$, infección del SNC en $16 \%$, cambio de drogas antiepilépticas en $16 \%$, epilepsia intratable en $11 \%$, hemorragia intracraneana en $11 \%{ }^{13}$. Otro trabajo con 141 niños con alteración de conciencia, $16 \%$ tuvo EENC, 48\% tuvo lesiones estructurales del SNC, $22 \%$ lesiones no estructurales, $13 \%$ epilepsia, $43 \%$ sin condición neurológica preexistente $^{21}$. Investigaciones en adultos han mostrado similares etiologías ${ }^{20}$.

En relación con los estudios de imágenes, destaca la importancia que tienen en la precisión del diagnóstico, ya que en 55,5\% de los pacientes se encontró una alteración responsable o contribuyente del EENC. Esto es fundamental ya que la etiología del EENC determina el pronóstico de estos pacientes ${ }^{24}$.

La detección precoz de EENC es importante para iniciar tratamiento dado el daño neuronal que se produce por las descargas eléctricas, evidenciada por otros por el aumento de la enolasa especifica de neuronas ${ }^{25}$.

La duración del EENC predice el pronóstico vital. Cuando el EENC dura 30 minutos la mortalidad reportada fue de $36 \%$ y si este dura más de 24 horas la mortalidad fue de $75 \%$.
Otras series reportan una mortalidad asociada al EENC de 51 a $57 \%$, pero es difícil separar el efecto del EENC de la patología subyacente ${ }^{11,22}$. Nuestra baja mortalidad (3\%) se puede explicar a que la mayoría de nuestros pacientes tuvo una etiología criptogénica y al manejo en UPC con EEG continuo.

Las secuelas encontradas en el seguimiento de estos pacientes se deben principalmente a etiología hipóxico-isquémica, destacando la parálisis cerebral y el retraso del desarrollo psicomotor. En relación a pronostico, a diferencia de lo que reportamos, los pacientes con EENC han tenido una evolución más favorable, debido probablemente al menor tiempo de seguimiento ${ }^{26}$.

Este estudio presenta las limitaciones propias de su diseño retrospectivo, además del sesgo producido por mantener a niños con factores de riesgo de EENC por más tiempo con monitoreo EEG.

En conclusión, la sospecha clínica, el antecedente de epilepsia y el apoyo con un EEG, especialmente prolongado, permite el diagnóstico oportuno y el tratamiento precoz de estos pacientes. El monitoreo EEG es una excelente herramienta para estos casos.

Dado las distintas etiologías en los pacientes es difícil evaluar un tratamiento estándar para el EENC.

Es importante realizar un estudio prospectivo que compare la evolución según la etiología y tratamiento realizado, además del pronóstico a largo plazo de estos pacientes.

\section{Referencias}

1.- DeLorenzo RJ, Hauser WA, Towne AR, et al: A prospective, population-based epidemiologic study of status epilepticus in Richmond, Virginia. Neurology 1996; 46 (4): 1029-35.

2.- Treatment of convulsive status epilepticus. Recommendations of the Epilepsy Foundation of America's Working Group on Status Epilepticus. JAMA 1993; 270 (7): 854-9.

3.- Meldrum BS, Brierley JB: Prolonged epileptic seizures in primates. Ischemic cell change and its relation to ictal physiological events. Arch Neurol 1973; 28 (1): 10-7.

4.- Proposal for revised clinical and electroencephalo- 
graphic classification of epileptic seizures. From the Commission on Classification and Terminology of the International League Against Epilepsy. Epilepsia 1981; 22 (4): 489-501.

5.- Walker M, Cross H, Smith S, et al: Nonconvulsive status epilepticus: Epilepsy Research Foundation workshop reports. Epileptic Disord 2005; 7 (3): 253-96.

6.- Kaplan $P W$ : Nonconvulsive status epilepticus. Semin Neurol 1996; 16 (1): 33-40.

7.- Cascino GD: Nonconvulsive status epilepticus in adults and children. Epilepsia 1993; 34 Suppl 1: S21-8.

8.- Salas-Puig J, Suárez-Moro R, Mateos V: Status epilepticus. Neurologia 1996; 11 Suppl 4: 108-21.

9.- Fernández-Torre JL, Gutiérrez-Pérez R, Velasco-Zarzosa M: Non-convulsive status epilepticus. Rev Neurol 2003; 37 (8): 744-52.

10.- Husain AM, Horn GJ, Jacobson MP: Non-convulsive status epilepticus: usefulness of clinical features in selecting patients for urgent EEG. J Neurol Neurosurg Psychiatry 2003; 74 (2): 189-91.

11.- Young GB, Jordan KG, Doig GS: An assessment of nonconvulsive seizures in the intensive care unit using continuous EEG monitoring: an investigation of variables associated with mortality. Neurology 1996; 47 (1): 83-9.

12.- Hosain SA, Solomon GE, Kobylarz EJ: Electroencephalographic patterns in unresponsive pediatric patients. Pediatr Neurol 2005; 32 (3): 162-5.

13.- Tay SK, Hirsch LJ, Leary L, Jette N, Wittman J, Akman $C I$ : Nonconvulsive status epilepticus in children: clinical and EEG characteristics. Epilepsia 2006; 47 (9): 1504-9.

14.- Jette N, Claassen J, Emerson RG, Hirsch LJ: Frequency and predictors of nonconvulsive seizures during continuous electroencephalographic monitoring in critically ill children. Arch Neurol 2006; 63 (12): 1750-5.

15.- Alehan FK, Morton LD, Pellock JM: Utility of electroencephalography in the pediatric emergency de- partment. J Child Neurol 2001; 16 (7): 484-7.

16.- Shneker BF, Fountain NB: Assessment of acute morbidity and mortality in nonconvulsive status epilepticus. Neurology 2003; 61 (8): 1066-73.

17.- Krumholz A, Sung GY, Fisher RS, Barry E, Bergey GK, Grattan LM: Complex partial status epilepticus accompanied by serious morbidity and mortality. Neurology 1995; 45 (8): 1499-504.

18.- Vespa PM, O'Phelan $K$, Shah M, et al: Acute seizures after intracerebral hemorrhage: a factor in progressive midline shift and outcome. Neurology 2003; 60 (9): 1441-6.

19.- Hauser WA: Status epilepticus: epidemiologic considerations. Neurology 1990; 40 (5 Suppl 2): 9-13.

20.- Claassen J, Mayer SA, Kowalski RG, Emerson RG, Hirsch LJ: Detection of electrographic seizures with continuous EEG monitoring in critically ill patients. Neurology 2004; 62 (10): 1743-8.

21.- Saengpattrachai M, Sharma R, Hunjan A, et al: Nonconvulsive seizures in the pediatric intensive care unit: etiology, EEG, and brain imaging findings. Epilepsia 2006; 47 (9): 1510-8.

22.- Abend NS, Dlugos DJ: Nonconvulsive status epilepticus in a pediatric intensive care unit. Pediatr Neurol 2007; 37 (3): 165-70.

23.- Alroughani R, Javidan M, Qasem A, Alotaibi N: Nonconvulsive status epilepticus; the rate of occurrence in a general hospital. Seizure 2009; 18 (1): 38-42.

24.- Santos-García D, Vázquez-Herrero F, Rodríguez-Osorio $X$, Prieto JM: Magnetic resonance imaging and epileptic status. Rev Neurol 2006; 43 (5): 309-10.

25.- DeGiorgio CM, Heck CN, Rabinowics AL, Gott PS, Smith T, Correale J: Serum neuron-specific enolase in the major subtypes of status epilepticus. Neurology 1999; 52( 4): 746-9.

26.- Aranguiz J, Hernández M, Escobar RG, Mesa T: Childhood epilepsy status in a university hospital: a descriptive study. Rev Neurol 2008; 47 (6): 286-90. 